\section{PSICOLOGÍA IBEROAMERICANA}

\section{Psicología lberoamericana}

ISSN: 1405-0943

psicología.iberoamericana@uia.mx

Universidad Iberoamericana, Ciudad de

México

México

Mendoza González, Brenda

Taller para la Detección de Casos de Abuso Sexual Infantil en Niños de Educación Básica

Psicología Iberoamericana, vol. 17, núm. 1, enero-junio, 2009, pp. 24-37

Universidad Iberoamericana, Ciudad de México

Distrito Federal, México

Disponible en: http://www.redalyc.org/articulo.oa?id=133912613004

- Cómo citar el artículo

Número completo

- Más información del artículo

Página de la revista en redalyc.org

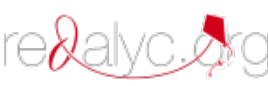

Sistema de Información Científica

Red de Revistas Científicas de América Latina, el Caribe, España y Portugal

Proyecto académico sin fines de lucro, desarrollado bajo la iniciativa de acceso abierto 
Psicología lberoamericana (Enero-junio, 2009). Vol. 17. No. 1.pp. 24-37 ISSN 1405-0943

\title{
Taller para la Detección de Casos de Abuso Sexual Infantil en Niños de Educación Básica
}

\author{
Workshop to Detect Sexual Abuse in Children of Elementary School
}

Brenda Mendoza González ${ }^{1}$

Secretaría de Educación Pública

\section{RESUMEN}

El objetivo del presente artículo es describir y explicar el Taller para la detección de casos de abuso sexual en niños de educación básica, diseñado para detectar abuso sexual en niños de cinco a quince años de edad, en estudiantes de escuelas públicas del Distrito Federal El programa es una propuesta proactiva, ya que también incide como preventivo, al proveer a los niños de herramientas que les permitirán identificar el comportamiento abusivo y revelar el suceso. El taller respaldó el diagnóstico de casos de abusos sexuales, ofreciendo sus resultados como prueba en procesos legales. Se diseñó con base en la aproximación cognoscitivo-conductual, los derechos de la niñez, y el currículo de la SEP; se estructura asimismo en cinco componentes: derechos de la niñez; maltrato; relajación; abuso sexual y comunicación asertiva.

Descriptores: Abuso sexual, detección de abuso sexual, programa de prevención, educación básica, educación sexual.

\section{ABSTRACT}

The aim of this work was to describe and explain a workshop entitled: Detection of child sexual abuse. This Workshop was designed to detect cases of sexual abuse in children with ages ranging between six to thirteen years old, for that the author carried out studies with one thousand children students of several public school of México City. The program is a pro active suggestion because give them abilities for the soon identification of abuse behavior and the disclosure. The program supported the sexual abuse diagnostic like an evidence in several legal process. Has a basis in Cognitive Behaviour approach and children rights and Education curriculum, too; has five components: Right children, Maltreatment, Relaxation, Sexual Abuse and Assertive Communication.

Key words: sexual abuse, sexual abuse detection, prevention program, elementary school children, sexual education.

\section{INTRODUCCIÓN}

El abuso sexual infantil es un problema que encaran las sociedades de todo el mundo; es un fenómeno complejo cuyas causas son una combinación de factores sociales, familiares e individuales, que afectan a niños de cualquier raza, estatus social o educativo (Whitaker, Le, Hanson, Baker, McMahon, Klein \& Rice, 2008).

La oms señala que actualmente hay tres millones de niños que sufren abuso sexual. Por su parte Save the children recientemente denunció a fuerzas armadas de la oNu por tener entre sus filas a integrantes que abusaban sexualmente de niños. En México, en investigaciones desarrolladas en la UNAM se ha identificado que son las niñas con una edad pomedio de 5.7 años, quienes se encuentran en mayor situación de riesgo (Serratos, 2003).

La investigación en abuso sexual infantil ha captado la atención de investigadores que desde las áreas experimental, educativa, social, clínica e incluso legal, 
han aportado información valiosa que ha ayudado para validar el testimonio de los niños que han sido víctimas (Yuille, 1989); se han ofrecido excelentes estudios para la elaboración de diagnósticos válidos y confiables (Hershkowitz, Fisher, Lamb \& Horowitz, 2007; Vázquez, 2003); se han diseñado técnicas en el área clínica para brindar ayuda a la víctima (Batres, 2000), y se han ofrecido herramientas de prevención diseñadas para los niños y para los padres (López, 2000), así como prácticas para seguir un proceso legal (Urra, 2002) y en general para ayudar a las víctimas en el proceso de revelación del suceso erradicando los mitos existentes en nuestra sociedad (Mendoza, 2002; Vázquez, 2003).

Actualmente los expertos en abuso sexual toman el concepto que ofreció The National Center on Child Abuse and Neglect (Martin \& Klaus,1978), que define al abuso sexual como los contactos e interacciones entre un niño y una persona mayor que él, quien utiliza al primero para estimularse él mismo, estimular al niño o a otra persona, situándose éste como el agresor (puede ser menor a 18 años), en una posición de poder o control sobre el menor; siendo dos características definitorias del abuso sexual: la desigualdad entre agresor y víctima (poder, edad, fuerza, etc.) y la utilización del menor como objeto sexual.

Los abusos sexuales pueden cometerse a través de tocamientos o sin ellos (exhibicionismo, proyección de imágenes, etc.), en muchos de los casos al no haber huellas identificables es más difícil probar que hubo abuso, sin embargo, actualmente existen instrumentos legales que han demostrado su eficacia para evitar que el abuso sexual no quede impune (Mendoza, 2004a; Vázquez, 2003).

El abuso sexual tiene efectos a corto y largo plazos identificables para su pronto tratamiento; Echeburúa y Guerricaechevarría (1998) identifican los efectos a corto plazos en el periodo evolutivo de la infancia como: pérdida de control de esfínteres, miedo generalizado, expresiones de agresión hacia otros, manifestaciones exhibicionistas, entre otras. Existen otras actitudes compartidas tanto en el periodo evolutivo de infancia y adolescencia como: problemas de sueño, cambios en la ingesta de alimentos, bajo rendimiento académico, emociones negativas (culpa, vergüenza, miedo); baja autoestima, depresión, ansiedad, rechazo a su cuerpo, desconfianza y rencor hacia los adultos, conocimiento sexual precoz e inapropiado para su edad; masturbación compulsiva, excesiva curiosidad sexual, retraimiento social. Los efectos a largo plazo que pueden exhibirse en la adultez son: depresión, ansiedad, intentos suicidas, consumo de drogas o alcohol, dificultad para expresar o recibir expresiones de cariño; fobias o aversiones sexuales, déficit en habilidades sociales.

Debido a los efectos a corto y largo plazos que el abuso sexual genera en los niños, y dado que generalmente no deja rastros en el cuerpo de las víctimas, se necesita el desarrollo de programas de detección que permita, en un ambiente educativo y de confianza ayudar a las víctimas a revelar episodios abusivos.

El objetivo del presente artículo es presentar la estructura general y los componentes que conforman un taller diseñado para la detección de casos de abuso sexual infantil. Se diseñó con base en la aproximación cognoscitivo-conductual (Bond \& Dryden, 2002), respaldado en la Ley de los derechos de las niñas, niños y adolescentes, así como en el currículo de educación básica; ha demostrado ser una herramienta eficaz en la identificación de niños y niñas abusados sexualmente, además de proveerlos de información que les permita reconocer comportamientos abusivos (no necesariamente sexuales), asimismo, les brinda habilidades que les permita identificar a una persona en la que podrán confiar y pedir ayuda en caso de afrontar alguna situación de abuso.

El programa fue diseñado y aplicado sistemáticamente por la autora para realizar investigaciones en casos de abuso sexual perpetrados en planteles escolares, casos que fueron atendidos durante su estancia por un grupo de especialistas en atención en casos de maltrato y abuso sexual (SEP). Su aplicación se hizo a 1025 niños y niñas de educación básica, con edades entre cinco y 15 años de edad, y sus resultados fueron aportados y aceptados como evidencia en procesos legales.

\section{MÉTODO}

El programa se impartió en más de 25 escuelas de educación básica del Distrito Federal, como parte de una estrategia sistemática para atender e investigar casos 
de abuso sexual reportados a la Administración Federal de Servicios Educativos para el D. F. La ruta de atención se muestra en la figura 1. la escuela o fuera de ella? (describir el nombre de la persona, parentesco, lugar, frecuencia); 2) ¿has vivido algún abuso sexual (describir el nombre de la persona, parentesco, lugar, frecuencia)?; 3) en caso de haber

Figura I. Ruta de atención de casos de abuso sexual (Administración Federal de Servicios Educativos del Distrito Federal)

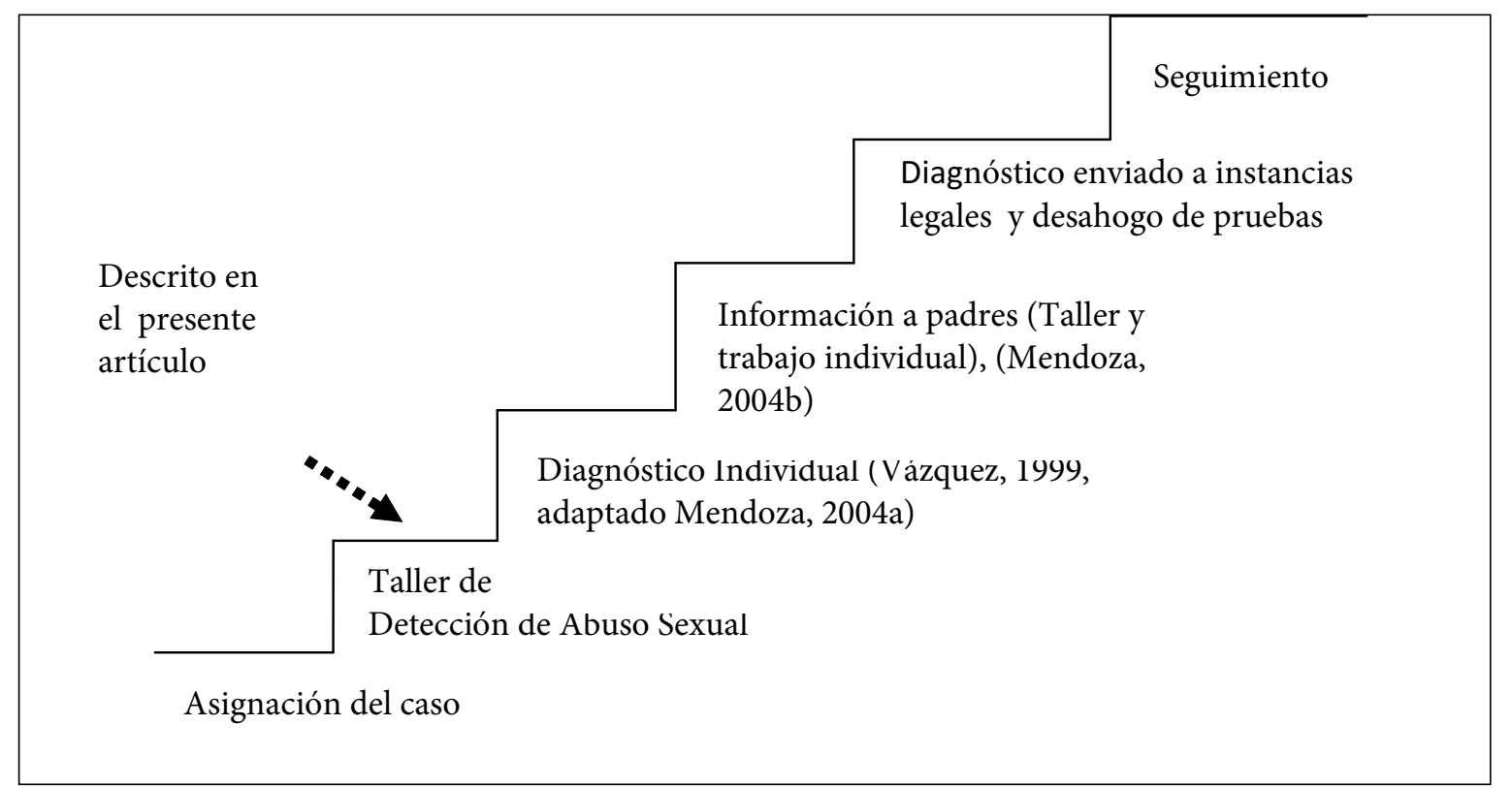

\section{Participantes}

Participaron 1025 alumnos de educación básica. El programa se aplicó en 25 escuelas del Distrito Federal (tres de nivel preescolar; 17 primarias y cinco secundarias).

Participantes: 20 niños de cinco años de edad (14 niñas y 6 niños), 245 niños de secundaria (128 chicas, 113 chicos), y 760 alumnos de primaria (397 niñas, 363 niños). Los alumnos se encontraban inscritos en escuelas públicas de las delegaciones: Álvaro Obregón, Azcapotzalco, Cuajimalpa, Gustavo A. Madero, Iztapalapa, Miguel Hidalgo, Tláhuac, Tlalpan, Venustiano Carranza.

Es importante señalar que en todos los casos los padres y las autoridades escolares dieron la autorización para impartir el taller.

\section{Instrumentos}

Se empleó un autorreporte con tres preguntas generales: 1) ¿has vivido algún tipo de maltrato dentro de contestado sí, nombra a las personas a quienes les hayas contado sobre el abuso o maltrato.

\section{Escenario}

El taller se impartió en un aula proporcionada por autoridades escolares, que tuviera iluminación, ventilación adecuada, así como mesas y sillas movibles en el caso de preescolar y sillas individuales o pupitres para el estudio en primaria y secundaria.

\section{Procedimiento}

En todos los casos, el taller se aplicó como una de las primeras estrategias de atención en escuelas dónde se había denunciado algún posible episodio de maltrato o abuso sexual ocurridos en el escenario escolar; generalmente el denunciante era la madre de la víctima.

Se establecieron diferencias en la aplicación del taller que obedecían a la edad y nivel escolar de los estudiantes. Con respecto a los niños en edad preescolar, se trabajó 
siempre en grupos pequeños máximo de cinco miembros; los participantes fueron los alumnos "riesgo" (es decir niños que probablemente se encontraban involucrados en situaciones de maltrato o abuso sexual). Conviene destacar que el material fue el mismo para todos, sin modificarse el lenguaje, la cantidad de los cantos y juegos (es decir, en edad preescolar se realizaban más cantos y juegos que incluso los propios niños compartían, a diferencia de los estudiantes de secundaria, en donde el único juego que se realizaba era el juego de distención canastas de frutas).

\section{Estructura del taller}

El Taller se conforma por cinco componentes: Derechos de los niños, niñas y adolescentes, maltrato, relajación; abuso sexual y comunicación asertiva organizadas en dos sesiones con duración total de cinco horas. La primera sesión contiene los primeros tres componentes y la segunda se integra por los dos últimos.

\section{Características generales del programa}

Es necesario que al inicio del taller se señalen reglas de convivencia (respeto mutuo); reglas de comunicación (comunicación asertiva: respetar turnos, escuchar, hablar en primera persona: yo pienso, yo creo, hacer contacto visual), y reglas de confidencialidad (respeto a la intimidad del alumnado). Se sugiere que el mobiliario durante las sesiones se organice en círculo; el especialista deberá colocarse a la misma altura visual que los niños. El taller deberá iniciar con una fase de rapport, que tendrá como objetivo establecer las bases de comunicación, confianza entre el grupo y el profesional que imparte el Taller.

\section{El primer componente hace referencia a los derechos de los niños y las niñas introducido bajo el nombre de: Mis derechos}

El objetivo del primer componente es que los niños y niñas tengan información del derecho que tienen de ser respetados física, sexual y emocionalmente.

En este componente se explica que personas de todos los países incluido México, se han reunido para decretar leyes que establezcan derechos (tienen derecho a jugar, a estar bien cuidados, e ir a la escuela) y obligaciones para la niñez (respetar a las personas con las que conviven y cumplir con tareas escolares, entre otras). Se sugiere que se empleen dinámicas que han sido desarrolladas por Amnistía Internacional para la educación en derechos humanos, las cuales están diferenciadas por grupo de edad, por lo que se pueden elegir dependiendo justamente de la edad de los niños con los que se trabaja (Amnistía Internacional, 2000).

La gran mayoría de los países han mostrado su preocupación por proteger a las niñas, niños y adolescentes del maltrato incluyendo el abuso sexual, por lo que han aceptado leyes internacionales como las establecidas en la Convención Internacional de los Niños, que en su Artículo 34 señala que se debe de proteger a los niños contra toda forma de abuso sexual; el Artículo 19 señala que se debe proteger a los niños contra toda forma de violencia; el Artículo 13 se refiere al ambiente escolar, y señala que en las escuelas, los maestros serán responsables de evitar cualquier forma de maltrato o abuso por miembros de la comunidad educativa, o entre el alumnado (bullying). ${ }^{2}$

\section{El segundo componente corresponde al tema de maltrato infantil introducido bajo el nombre de: Vayamos definiendo}

Este componente busca a través de fomentar un dialogo franco y abierto entre especialista y niños, que conjuntamente construyen la definición de maltrato infantil y con ello identifican los diferentes tipos de maltrato incluyendo al abuso sexual en los diferentes escenarios en los que se puede presentar (por ejemplo casa, escuela, ocio).

Durante esta sesión a través de una dinámica conocida como "lluvia de ideas" los niños generalmente definen el concepto de maltrato con experiencias propias o de otros. Se sugiere hacer uso de la guía de preguntas diseñadas especialmente para este componente (cuadro 1), conviene destacar que la guía de preguntas se adaptaba a la edad de los niños con los que se trabajaba. El especialista concluirá la actividad cuando los niños y niñas hayan identificado las tres formas de maltrato infantil: físico, verbal y sexual.

${ }^{2}$ Un alumno o grupo de alumnos dirigen constantemente maltrato hacia otro alumno, regidos por desequilibrio de poder y por la ley del silencio. 
El tercer componente corresponde a técnicas de relajación, introducido bajo el nombre de: Un respiro y nos vamos

Este componente tiene por objetivo que los niños conozcan habilidades de relajación que puedan emplear en su vida diaria para afrontar situaciones de estrés. Primero, se les enseña a respirar correctamente. Segundo, se les adiestra en la aplicación de la técnica tensión-relajación (contraer y soltar los músculos de cada una de las partes de su cuerpo) (Cautela \& Groden, 1989); esta técnica fue adaptada a la edad de los niños con niños de preescolar y primaria se usaba la analogía de "soldados de plomo" para tensar músculos, y "muñecos de trapo" para relajarlos, con estudiantes de segundo de primaria hasta secundaria se emplean nú- meros que se asignan al estado de tensión y relajación (4 es muy tenso, 3 es tenso, 2 disminuye la tensión, 1 relajación). La técnica de tensión-relajación se puede emplear prácticamente en cualquier lugar, auxiliándoles en situaciones de tensión, enojo o cuando quieran concentrarse con mayor facilidad en sus tareas académicas.

\section{El cuarto componente corresponde al manejo} del abuso sexual, introducido bajo el nombre de: Yo pienso, yo opino, y me cuido

Debido a la complejidad de este comportamiento, el componente abuso sexual, se estructura en varias actividades, y cada una de ellas va acompañada de una guía de preguntas.

\section{Cuadro I. Guía de preguntas sugeridas por el especialista en abuso sexual y cuyo orden va de lo general a lo específico}

\subsection{Preguntas que abren la discusión}

¿Qué hacen los adultos que te cuidan cuando se enojan contigo? ¿Cómo te castigan? ¿Qué hace papá o mamá cuando no los obedeces? ¿Crees que está bien la forma en la que te tratan cuando se enojan o te regañan? ¿Cómo te sienten cuando te pegan? ¿Conoces al "zapato volador"?

\subsection{Introducción del término maltrato}

Cuando los niños o niñas introducen el término de maltrato se sugiere que se pregunte al grupo:

¿Qué creen que sea el maltrato infantil?

¿Cómo crees que se maltrata a los niños o a las niñas? ¿Alguien quiere compartir un ejemplo de maltrato infantil? ¿Qué objetos usan para pegarles? ¿Cómo te gustaría que se comportaran tus papás cuando no los obedeces?

\subsection{Introducción del término maltrato emocional} Generalmente los niños empiezan por identificar al maltrato físico como maltrato infantil; sin embargo, el especialista deberá guiar la sesión para que los niños sean capaces de identificar a otras formas de maltrato como el emocional o el sexual. Las preguntas que puede emplear son:

Además de pegarle a un niño; ¿de qué otra forma se le puede lastimar? ¿Se puede lastimar a un niño sin pegarle?

¿Creen que la única forma de maltratar a un niño es pegándole? ¿Se puede maltratar con palabras? ¿Rechazar o ignorar es una forma de maltrato?

\subsection{Introducción del término abuso sexual}

Para que los niños identifiquen que el abuso sexual es una forma de maltrato puede preguntárseles: Además de lastimar a un niño o niña física o emocionalmente ¿de qué otra forma creen que se le puede maltratar?

¿Se puede maltratar el cuerpo de un niño o niña sin que existan golpes? ¿Creen que alguna persona puede tocar el cuerpo de un niño o niña sin que el niño o niña quiera? ¿Alguien puede explicar por qué? 


\section{Primera actividad: el cuerpo humano y sus cuidados (para guía de preguntas véase el cuadro I)}

En esta actividad los niños identificarán que su cuerpo les pertenece y que sólo ellos pueden decidir en función de él; sin embargo, aprenderán también que será necesario que en ocasiones algunos adultos revisen su cuerpo para mantenerlo sano y que existen normas de seguridad que regulan el tocamiento del cuerpo de las personas (por ejemplo: cambiar pañal, revisión medica, higiene, etcétera).

Durante la actividad los niños conocerán que es natural y sano llamar a los genitales por su nombre: "pene, vulva, ano, senos", aprendizaje que se realiza a través de esquemas educativos del cuerpo tanto de niños como de niñas (se adecúan a la edad de los niños o adolescentes), incluso esta lámina se empleó con niños de secundaria, observándose que facilitaba el trabajo con ellos para nombrar sin tabúes cada una de las partes de su cuerpo.

Se explica también la importancia de la higiene y el cuidado de su cuerpo, cuidarlo de golpes, de caídas, pero también de caricias o tocamientos que ellos o ellas no quieren que les hagan. En esta actividad también se tendrá que aportar una visión positiva de la sexualidad infantil, en la que identifiquen conductas que se manifiestan alrededor de los tres y doce años de edad que son totalmente naturales y que ayudarán a combatir muchos de los mitos que desafortunadamente se tienen en México.

\section{Segunda actividad: lo público y lo privado}

El objetivo es que los niños identifiquen las actividades que la sociedad ha determinado como públicas y privadas (por ejemplo lavarse las manos, nadar, o ir al baño), siempre observando esta diferencia como algo natural, sin tabúes.

El especialista comenzará la sesión con una asamblea, en la cual los niños identificarán actividades y partes públicas y privadas de su cuerpo (para guía de preguntas véase el cuadro 2). Durante la actividad se identificarán las actividades que se pueden realizar en público o en privado justamente. El especialista expresará al grupo que las actividades privadas son aquellas que involucran a las partes íntimas, por ejemplo, cambiarse de ropa interior (lo que implica descubrir las partes íntimas); sin embargo, no por ser privada signi- fica que es algo sucio, o algo de lo que no se pueda hablar, simplemente quiere decir que cualquier parte del cuerpo, pero principalmente las privadas, no deben de ser tocadas por nadie sin el consentimiento del niño o de la niña. Con estudiantes de secundaria se abordan la importancia de lo público y lo privado con el objetivo de que los jóvenes afirmaran su derecho a tener momentos de privacidad, su derecho a conocer su cuerpo e incluso tocarlo; con los estudiantes de secundaria se abordaba un tema tabú: la masturbación, en la que se hablaba de los mitos en torno a ella, y de su decisión a ejercerla o no bajo los parámetros de privacidad e higiene.

\section{Tercera actividad: ¿qué es el abuso sexual?}

Esta actividad tiene por objetivo que los niños identifiquen la existencia del abuso sexual, así como a las personas que pueden llegar a cometerlos (familiares, vecinos, amigos, algún cuidador, etc.); el lugar en donde pueden llegar a cometerse (casa, escuela, etc.), $\mathrm{y}$ los tipos de abusos sexuales (caricias, tocamientos).

La actividad inicia explicando que existen tocamientos adecuados y otros que no lo son, y que tienen la posibilidad de identificarlos, ya que los adecuados los hacen sentir bien, les dan tranquilidad (el beso en la frente), en cambio los tocamientos inadecuados les hacen sentir miedo, vergüenza, o confusión, por lo que deberán comunicar inmediatamente a un adulto de confianza cómo se sintieron.

El especialista comentará que nadie tiene derecho a tocar o acariciar sus partes íntimas por medio de amenazas, chantajes o infundiéndoles miedo o lastimándoles. El especialista puede emplear preguntas como: ¿permitirías que alguien te pique tu ojito?, generalmente responden que no, por lo que se puede argumentar: "de igual manera nadie tiene derecho a acariciarte, o tocarte de manera desagradable".

Por medio de una dinámica de "lluvia de ideas" y con el uso de marionetas se pregunta al grupo sobre sus creencias acerca del abuso sexual infantil; el especialista realizará un resumen. Al término de este componente se recomienda realizar un juego de distención que permita que los niños se rían, se relajen, griten y se muevan, los juegos se clasifican por edad, de tal forma que el especialista previamente elige cuál de los juegos puede adecuarse a la edad de los niños con los que trabajará (Cascón \& Martín, 2000). 


\section{Cuadro 2. Guía de preguntas sugeridas por el especialista para identificar actividades públicas y privadas}

\begin{tabular}{|l|l|}
\hline Apertura de dinámica & $\begin{array}{l}\text { "Si por ejemplo una persona se lava la cara:¿qué partes del } \\
\text { cuerpo esta limpiando?". } \\
\text { ¿La cara es una parte del cuerpo pública o privada?, ¿Por } \\
\text { qué? } \\
\text { Entonces, ¿lavarse la cara es una actividad pública o pri- } \\
\text { vada?”. }\end{array}$ \\
\hline Actividad: bañarse & $\begin{array}{l}\text { ¿Es una actividad pública o privada?, ¿Qué partes del } \\
\text { cuerpo involucra, públicas o privadas? }\end{array}$ \\
\hline Actividad: nadar & $\begin{array}{l}\text { ¿Es una actividad pública o privada? ¿Qué partes de su } \\
\text { cuerpo cubren cuando nadan? ¿Por qué creen que se cubre } \\
\text { el pene en el caso de los niños y la vulva y el pecho en el } \\
\text { caso de las niñas? }\end{array}$ \\
\hline Los niños analizan, reflexionan, concluyen; el especialista participa como guía. \\
\hline
\end{tabular}

\section{Cuarta actividad: el secreto}

Esta actividad tiene como objetivo que el niño identifique la función del secreto en los casos de abuso sexual. En esta sesión comienza con un cuento diseñado para este componente, cuyo objetivo es demostrar a los niños que pueden platicar a sus padres alguna situación difícil, que a veces no cuentan por miedo a ser culpados, castigados, etc. (Mendoza, 2002), puede contarse algún otro cuento cuyo mensaje sea: decir la verdad, aun cuando sientan miedo, vergüenza, o culpa. Al finalizar el cuento, el especialista expresa que el agresor quiere que guarden el secreto para seguir comportándose abusivamente con ellos sin que nadie se entere, por lo tanto hay que romper el silencio y pedir ayuda lo mas pronto posible. Este cuento fue empleado con todos los niños de todas las edades, debido a que el cuento fue muy bien aceptado por los estudiantes de secundaria.

\section{Quinta actividad: ¿y quién tiene la culpa?}

Esta actividad tiene por objetivo que los niños identifiquen que en casos de maltrato y abuso sexual ellos no son responsables del comportamiento del agresor, y que es natural que surjan emociones como la culpa, el miedo, la vergüenza, la tristeza, etcétera.

A través del empleo de marionetas, el especialista expresará que en casos de abuso sexual los niños tienen miedo de contar la experiencia ya que el agresor emplea amenazas, chantajes o les infunde miedo; o bien, no hablan de ello porque les da vergüenza hablar de sus partes íntimas, o tienen la falsa creencia de que ellos son culpables de lo que pasó. A través de una dinámica de "lluvia de ideas" (con niños de preescolar y primero de primaria se emplearon marionetas) se identifica la función de: 1) las amenazas en episodios de abuso sexual, identificándose que se emplean para evitar que el niño revele a otros adultos la agresión, 2) el secreto, identificándose que también tiene la función de evitar que el abuso se descubra, para que siga ocurriendo cada vez que el agresor lo determine, 3) la pertenencia de su cuerpo, así como la naturalidad con la que hay que hablar de las partes íntimas (tendrá que compartirse la idea de la sexualidad infantil positiva, combatiendo los mitos mas generalizados en México en torno a la sexualidad).

\section{Sexta actividad: rompiendo el silencio}

Esta actividad tiene por objetivo que los niños conozcan la importancia de la revelación del abuso sexual, e identifiquen, por otro lado, a la persona de confianza que pueda ayudarlos en caso de que requieran ayuda no sólo en casos de agresión sexual. En esta sesión se explica que cuando un niño revela que ha sido abusado sexualmente, se le debe creer, y que además se le debe de reconocer su valentía por haberlo dicho, considerando que es natural que se tenga miedo o vergüenza de 
contar una experiencia así, ya que incluso puede sentir confusión, pero la única forma de ayudarlo es cuando se decide a romper el silencio. En esta actividad se invita a identificar a una persona en la que puedan confiar, haciendo uso de una hoja de trabajo definida para ello (para guía de preguntas véase cuadro 3), con los niños de preescolar y primero de primaria, se realiza el ejercicio en forma de entrevista.
El cuarto componente corresponde a la comunicación asertiva: $y$ si me da miedo... ¿cómo lo digo?

El objetivo del componente es que los niños desarrollen habilidades para expresar sentimientos y emociones, específicamente que aprendan a expresar de manera oral o escrita cuando otra persona les toca su cuerpo y no les gusta.

\section{Cuadro 3. Guía de preguntas para la identificación de un adulto que brinde ayuda y apoyo al}

niño o niña

\begin{tabular}{|c|c|c|c|c|c|}
\hline \multicolumn{6}{|c|}{$\begin{array}{l}\text { Puedo pedir ayuda a... } \\
\text { Escribe una palomita ( }(\checkmark) \text { a la persona que cumple } \\
\text { con la afirmación que está escrita a la derecha }\end{array}$} \\
\hline Afirmación & Mamá & Papá & Tío/a & Profesor/a & $\begin{array}{l}\text { Otra persona. Es- } \\
\text { cribe su nombre }\end{array}$ \\
\hline \multicolumn{6}{|l|}{$\begin{array}{l}\text { 1. Nunca ha tocado mis partes íntimas usando } \\
\text { amenazas, intimidaciones, golpes, etcétera. }\end{array}$} \\
\hline \multicolumn{6}{|l|}{ 2. Es una persona a la que quiero mucho. } \\
\hline \multicolumn{6}{|l|}{$\begin{array}{l}\text { 3. Esa persona me escucha cuando me siento } \\
\text { triste. }\end{array}$} \\
\hline \multicolumn{6}{|l|}{$\begin{array}{l}\text { 4. Cuando le cuento situaciones difíciles que } \\
\text { me han ocurrido me cree. }\end{array}$} \\
\hline \multicolumn{6}{|l|}{ 5. Siento que esa persona me quiere. } \\
\hline \multicolumn{6}{|l|}{ 6. Siento que me cuida. } \\
\hline \multicolumn{6}{|l|}{$\begin{array}{l}\text { 7. Siento que le interesa mucho lo que me } \\
\text { pasa. }\end{array}$} \\
\hline \multicolumn{6}{|l|}{ 8. Siento que puedo confiarle un secreto. } \\
\hline \multicolumn{6}{|l|}{ 9. Me ha ayudado cuando se lo he pedido. } \\
\hline \multicolumn{6}{|l|}{$\begin{array}{l}\text { 10. Me ha protegido cuando he sentido mie- } \\
\text { do. }\end{array}$} \\
\hline \multicolumn{6}{|l|}{ Número total de "palomitas". } \\
\hline \multicolumn{6}{|c|}{$\begin{array}{l}\text { Nombre de la persona que obtuvo mayor número de "palomitas" (requisito: haber cumplido con la afirmación nú- } \\
\text { mero 1) }\end{array}$} \\
\hline La persona en la que puedo confiar se llama: & & & & & \\
\hline
\end{tabular}


El especialista señalará que al expresar emociones (felicidad, enojo, tristeza, etc.) a otra persona pueden hacerlo de forma verbal o escrita, cuando se hace de forma verbal tendrán que seguir tres pasos: mirar a los ojos, expresar cómo se sienten, y manifestar la razón que les causa dicha emoción. Se señalará que en caso de que quieran confiar a alguien alguna situación que les haya provocado miedo, tristeza, angustia, deberán elegir a una persona de confianza (puede ser la persona que eligieron a través de la actividad mostrada en el cuadro 3) y contarle lo que le sucedió.

El especialista solicitó que a través de un autorreporte expresarán si habían vivido alguna situación de maltrato o abuso sexual (en caso de haber ocurrido, tendrían que especificar nombre del agresor, lugar, frecuencia); esta estrategia fue usada con niños de segundo de primaria y jóvenes de secundaria; con el alumnado de preescolar y primero de primaria se en las que se desarrollaría tal revelación (Mendoza, 2004b); lo anteriormente descrito corresponde a una segunda fase del procedimiento que por cuestiones de espacio no se describirá en este artículo (figura 1).

\section{RESULTADOS}

En la tabla 1 se muestran los resultados obtenidos a partir de la aplicación del taller a alumnos de educación básica.

En dicha tabla se observa que el alumnado identificó a 51 profesores que los agredieron con algún tipo de maltrato (emocional-físico, acoso o abuso sexual). Del total de profesores identificados; $80 \%$ de ellos se detectaron en el nivel primaria, $9.8 \%$ en jardín de niños y $10.2 \%$ secundaria.

El 55\% del total de los maestros ejercieron maltrato emocional y físico hacia sus alumnos, en todos los casos el profesorado exhibió ambos tipos de maltrato; $33.3 \%$

Tabla I. Distribución de la muestra por nivel de educación básica

\begin{tabular}{|c|c|c|c|c|c|c|c|c|}
\hline \multirow{3}{*}{ Grupos } & \multicolumn{8}{|c|}{ Nivel educativo } \\
\hline & \multicolumn{2}{|c|}{ Jardín de niños } & \multicolumn{2}{|c|}{ Primaria } & \multicolumn{2}{|c|}{ Secundaria } & \multicolumn{2}{|c|}{ Total } \\
\hline & $M^{*}$ & $\mathrm{~F}^{\star}$ & & $\mathrm{F}$ & M & $\mathrm{F}$ & M & $\mathrm{F}$ \\
\hline $\begin{array}{l}\text { Maltrato } \\
\text { Emocional-físico }\end{array}$ & 0 & 0 & 9 & 19 & 0 & 0 & 9 & 19 \\
\hline Acoso sexual & 0 & 0 & 1 & 0 & 5 & 0 & 6 & 0 \\
\hline Abuso sexual & 4 & 1 & & 0 & 0 & 0 & 16 & 1 \\
\hline Subtotal & 4 & 1 & 22 & 19 & 5 & 0 & 31 & 20 \\
\hline Total & & & & & & & & \\
\hline
\end{tabular}

${ }^{*} \mathrm{M}=$ Masculino, $\mathrm{F}=\mathrm{Femenino}$

realizó la actividad en forma de entrevista (los testimonios fueron grabados).

Cuando se detectaba algún caso de maltrato o abuso sexual, se procedía a desarrollar una entrevista individual con la víctima (analizada con índices que validan el relato infantil, Mendoza, 2004a), posteriormente se lograba la autorización del niño o la niña para revelar "el secreto" a los padres, lo cual se realizó en un ambiente de información, protección y apoyo, y donde el niño era libre de decidir las condiciones fue identificado como adultos que abusaron sexualmente de los niños (doce profesores, dos conserjes, y dos esposos de profesoras frente a grupo) $11.7 \%$ fue identificado como profesores que acosaban sexualmente a sus alumnos.

Con respecto al género, del total de profesores identificados, $60.7 \%$ fueron hombres y $39.2 \%$ mujeres; se identificaron más mujeres que ejercían maltrato emocional y físico hacia sus alumnos (37.2\%) que hombres (17.6\%) y en su mayoría fueron éstos quienes fueron 
identificados por el alumnado como profesores que abusaron sexualmente (31.3\%) más que las mujeres (1.9\%) o acosaron sexualmente a los alumnos (11.7 por ciento).

En los casos de abuso sexual infantil (después de culminado el taller), el alumnado aceptó revelar el episodio abusivo a una persona de confianza (siempre se tuvo la guía y el acompañamiento del especialista). Previo a la revelación del abuso sexual, los padres recibieron un taller de prevención y atención de abuso sexual infantil, los contenidos de dicho taller se derivaron del trabajo aquí presentado. A partir de los resultados obtenidos de los talleres, se solicitó la intervención de instancias jurídicas de la SEP o de contraloría interna (en su caso), asimismo se extendió una invitación a los padres de familia para ejercer su derecho a realizar una denuncia legal (en instancias fuera de la SEP).

Tabla 2.Tipos de conductas que se exhibieron en cada tipo de maltrato, en cada nivel educativo

\begin{tabular}{|c|c|c|c|}
\hline & \multicolumn{3}{|c|}{ Nivel educativo } \\
\hline Grupos & Jardín de niños & Primaria & Secundaria \\
\hline $\begin{array}{l}\text { Maltrato } \\
\text { emocional }\end{array}$ & & $\begin{array}{l}\text { Burlas } \\
\text { Apodos, insultos } \\
\text { Impide participar } \\
\text { Amenazas } \\
\text { Bajar los pantalones del niño } \\
\text { frente al grupo } \\
\text { Hablar mal del alumnado que no } \\
\text { está presente }\end{array}$ & \\
\hline Maltrato físico & & $\begin{array}{l}\text { Golpear con objetos } \\
\text { Golpear en partes del cuerpo } \\
\text { Jalones, empujones, pellizcos } \\
\text { Maltrato a pertenencias } \\
\text { Mantener a los alumnos de pie o } \\
\text { hincados durante la clase } \\
\text { Mojarles la cabeza y ponerlos al } \\
\text { sol }\end{array}$ & \\
\hline Acoso sexual & & $\begin{array}{l}\text { Invitación a un acto sexual } \\
\text { Pláticas (contenido sexual) } \\
\text { Preguntas insistentes sobre la } \\
\text { sexualidad de los alumnos }\end{array}$ & $\begin{array}{l}\text { Invitación (comportamiento sexual) } \\
\text { Pláticas (contenido sexual) } \\
\text { Miradas al cuerpo de los alumnos } \\
\text { Intentos para tocar el cuerpo de los } \\
\text { chicos } \\
\text { Preguntas insistentes sobre la sexua- } \\
\text { lidad de los alumnos }\end{array}$ \\
\hline Abuso sexual & $\begin{array}{l}\text { Tocamiento (par- } \\
\text { tes íntimas) } \\
\text { Amenazas } \\
\text { Obligación a } \\
\text { mantener el } \\
\text { secreto } \\
\text { Coerción }\end{array}$ & $\begin{array}{l}\text { Tocamiento (partes íntimas) } \\
\text { Amenazas } \\
\text { Obligación a mantener el secreto } \\
\text { Coerción }\end{array}$ & \\
\hline
\end{tabular}


En la tabla 2 se presenta el tipo de conductas que presentaron los adultos y que fueron identificadas por el alumnado. Se indica asimismo que el profesorado que exhibe maltrato emocional emplea conductas asociadas a la exclusión y rechazo (impide participar, habla mal de un alumno cuando no está, etc.); intimidación (amenazar), humillación (pasar a un alumno al frente del grupo y bajarle los pantalones), y maltrato verbal (burlas, insultos, apodos); se identificó que en el tipo de maltrato físico existen conductas que permiten el uso de objetos para pegar (gis, borrador, regla), o el uso de alguna de las partes del cuerpo para pegar (puñetazo, patada), maltrato a las pertenencias del alumnado (tirar a la basura cuadernos, arrancar hojas para desaprobar un trabajo, etc.), y conductas violentas (mojar la cabeza de un alumno y ponerlo bajo los rayos del sol).

Con respecto a las conductas de abuso sexual, se identificó que en todos los casos el adulto empleó amenazas (si dices algo te repruebo; si dices algo ya no te voy a querer).

A continuación se muestra el número de denuncias con respecto a las delegaciones políticas en el Distrito Federal recibidas en la SEP y cuyas investigaciones se describieron en el presente artículo: delegación Iztapalapa:14 denuncias; Álvaro Obregón: 4 denuncias; Tláhuac: 3 denuncias; Gustavo A. Madero: 2 denuncias; Venustiano Carranza: 2 denuncias; Tlalpan: 2 denuncias; Azcapotzalco: 2 denuncias; Miguel Hidalgo: 1 denuncia y Cuajimalpa: 1 denuncia.

\section{CONCLUSIONES}

El taller aquí presentado se integra dentro de un programa de educación sexual que permite a los niños identificar comportamientos abusivos, al tiempo que les habla sobre los aspectos positivos de su sexualidad, asumiendo las manifestaciones sexuales a lo largo del desarrollo de su vida (juego sexuales) como parte natural de su desarrollo (López, 2000).

A través de los resultados obtenidos, se concluye que el taller es una herramienta eficaz para la identificación de comportamiento de maltrato y abuso por parte de miembros de la comunidad educativa (profesorado, conserjes, etc.), facilitando la descripción del agresor y el lugar de los hechos, así como la revelación del suceso a un familiar de confianza. Particularmente, en casos de abuso sexual, se vuelve indispensable contar con herramientas eficaces y económicas que permitan la identificación temprana de víctimas de maltrato infantil, evitando que éste se vuelva crónico reduciendo así las consecuencias que afectan el desarrollo evolutivo de los niños, y que tiene efectos importantes en la salud como ansiedad, depresión, miedo y desconfianza a los hombres (Echeburúa \& Guerricaechevarría, 1998).

Otro de los objetivos del taller fue que las víctimas ejercieran su derecho de ser protegidas por adultos, el cual asumieron al aceptar revelar a una persona de confianza, el comportamiento abusivo del que fueron víctimas, este objetivo se logro a través de los componentes contenidos en el taller, especialmente el que refiere a los derechos de la infancia, tema transversal (así determinados en el currículum de la SEP), que enfrenta serias dificultades en algunas escuelas tradicionales, principalmente debido al miedo que expresan algunos profesores para ponerlo en práctica, ya que consideran que de este modo se les quita poder como profesorado (Cárdenas, 2003).

Otra conclusión es la que permite reconocer que el taller es una herramienta que posibilita el reconocimiento de que el uso de la violencia debe ser rechazado, para lo cual primero hay que reconocerlo. Este reconocimiento permitió a los niños describir en los autorreportes las conductas de maltrato y abuso que algunos miembros de la comunidad educativa habían ejercido hacia ellos. Conviene destacar que el contenido del taller proporcionado al alumnado se compartió con los padres de familia de los niños que fueron víctimas de abuso sexual, lo que evitó que padres hicieran una doble victimización a sus hijos, al brindarles su apoyo (creyendo en ellos); la prevención de abuso sexual infantil debe hacerse a nivel primario y no en el nivel terciario como aquí se realizó, práctica que se ha venido promoviendo a través de la difusión de la escuela para padres (Ayala, Carrancedo, Hubard, Marin, Moreno, Pérez \& Santín, 2000), en la que se pueden incluir múltiples temas para mejorar la calidad de la comunicación y confianza entre padres e hijos, requisitos indispensables para reducir el riesgo de que los niños sean abusados sexualmente.

Por otra parte, los resultados permitieron identificar que el profesorado que ejerció maltrato emocional, también lo hizo en el aspecto físico; estos resultados 
pueden explicarse con base en la Teoría de Coerción de Patterson, quien describe que una de las posibilidades para exhibir maltrato infantil es cuando el adulto ejerce la violencia que escala en intensidad en la medida que la víctima no muestra algún comportamiento que detenga el abuso del adulto, es decir, se somete de tal forma que refuerza las futuros ataques y el escalamiento del comportamiento coercitivo (Patterson, 1982).

Los resultados permitieron identificar que el tipo de maltrato ejercido se diferenció por el nivel escolar. En el nivel preescolar se identificó a alumnos que reconocieron haber vivido episodios de abuso sexual; en el nivel de secundaria algunos jóvenes aceptaron haber sido víctimas de acoso sexual, mientras que en el nivel de primaria, se identificaron tres tipos de abuso: maltrato mixto (emocional y físico), acoso y abuso sexual, estas diferencias pueden explicarse debido a que las etapas de mayor vulnerabilidad depende del periodo evolutivo de los niños, algunos autores refieren que las edades de mayor riesgo para vivir abuso sexual se encuentran entre los 6 y 12 años edad (Finkelhor, 2005), con respecto al acoso sexual se identificó que los niños de secundaria de esta muestra se encuentran en mayor riesgo de vivirlo, riesgo que se explica debido a que comienzan a aparecer cambios físicos y desarrollo sexual, al tiempo que aún son personas fácilmente manipulables (López, 2000).

Con respecto al género en los adultos identificados, los resultados permiten identificar que fueron hombres quienes ejercieron mayor abuso sexual, $y$ en general mayor maltrato hacia el alumnado, resultados que pueden explicarse con hallazgos derivados de estudios que señalan que uno de los riesgos de ejercer la violencia no se asocia al sexo biológico, si no más bien a las conductas asociadas a los estereotipos tradicionales femeninos o masculinos, mostrando mayor riesgo los hombres debido a la asociación al papel dominante al que se le ha conferido (machismo), incluso la aceptación social del uso de la violencia (Saldivar, Ramos, \& Saltijeral, 2004; Young \& Sweeting, 2004).

En lo que refiere a las delegaciones políticas, se identificó que la demarcación de Iztapalapa fue la que recibió un mayor número de denuncias. Cabe aclarar que de ninguna manera se puede establecer algún tipo de relación causal con respeto al nivel económico o educativo y el riesgo de vivir maltrato, sin embargo, se podría hipotetizar (partiendo de lo identificado en otros estudios), que los niños involucrados en estas denuncias tenían menos factores que pudieran protegerlos del riesgo de sufrir maltrato, como vivir en un ambiente familiar libre de abuso o ser constantemente monitoreadas por los padres (conocer a su grupo de amigos, su comportamiento escolar, etc.), y contaban con mayores factores de riesgo, como la falta de información que existe sobre al abuso sexual, debido a que muchas veces existe el tabú de tener acceso a ésta, incluyendo la etapa infantil (Whitaker et al., 2008), desafortunadamente como señalan algunos investigadores, actualmente la comunicación entre padres e hijos sobre la prevención de abuso sexual, es aún escasa (Del Campo \& López, 2006).

A través del taller se identificó que aún existen profesores que emplean prácticas de disciplina coercitivas para tratar de establecer hábitos en el alumnado, actitudes que lastiman la confianza y comunicación en la relación profesor-alumno, y que impide la construcción de aulas inclusivas. En la escuela se puede establecer y desarrollar el aprendizaje de comportamiento prosocial, así como el establecimiento de los derechos $\mathrm{y}$ obligaciones, que pueden practicarse a diario en las aulas escolares, por medio del modelo de comportamiento del principal agente de cambio en el contexto escolar: el profesorado, sin embargo, difícilmente se podrá enseñar cómo rechazar la violencia en tanto la plantilla de docentes en escuelas tradicionalistas empleen prácticas de disciplina coercitiva (prohibidas en el Artículo 42 de la Ley General de Educación). Lamentablemente, no es una novedad el que existan profesores que ejercen prácticas de disciplina inconsistente acompañadas de maltrato hacia los alumnos. En una consulta infantil y juvenil se identificó que alumnos de seis a nueve años de edad señalaron como una de sus principales preocupaciones la violencia de la que son objeto, en especial son vulnerables a los golpes y regaños que reciben del profesorado; en esta misma consulta los alumnos de 12 a 15 años de edad identificaron como uno de sus principales problemas el enfrentar la falta de información sexual (Nateras, Torres \& Linares, 2004).

A partir del supuesto de que los seres humanos nos encontramos en situaciones de elección, se debe ayudar a los niños a tomar decisiones con base en la educación. El programa de detección que aquí se presentó 
permitió a los niños adquirir información objetiva para reconocer de la existencia de conductas abusivas (requisito indispensable para su erradicación), así como los cursos de acción que pueden seguir para solicitar ayuda.

A lo largo de las investigaciones que se llevaron a cabo en las escuelas públicas, se pudo constatar la urgente necesidad de contar con un programa de prevención e intervención sistemático, evaluable, que se aplique en el proceso educativo cotidiano (habilidades de comunicación asertiva, respeto y cuidado hacia el cuerpo) a alumnos de educación básica con el objetivo de reducir la incidencia de la problemática a través de la educación, así como la identificación temprana de casos de abuso sexual infantil.

La investigación desarrollada para la identificación de casos de abuso sexual ocurridos en el contexto escolar (figura 1) permitió también constatar que aún existe desconocimiento en algunos miembros de la comunidad educativa con respecto a la manera de actuar en casos de abuso sexual, ya que desafortunadamente en algunos de los casos, este déficit llevó a producir una doble victimización, que dificultó el proceso de revelación, y sólo provocó estigmatizar a las víctimas y sus familiares.

El taller aquí presentado es un programa que deriva de la investigación puente o translational, el cual se define como una línea colaborativa entre la investigación básica y aplicada, cuya finalidad es el desarrollo de propuestas para lograr cambios sociales importantes (Lerman, 2003), como es el caso de la prevención e intervención de episodios de abuso sexual, problemática cuyos costos económicos y sociales afectan a la familia y la escuela y por ende la comunicación entre ellas, además de que impactan gravemente a la sociedad.

El estudio del acoso escolar a partir de la investigación puente, permitirá fundamentar y esclarecer nuevas preguntas de investigación para ser analizadas en el área básica o aplicada

Finalmente, a pesar de que el programa se ofreció y se aprobó como evidencia empírica en el proceso legal de los casos de abuso sexual identificados, no se ofrecen medidas pre y post que pudieran aportar evidencia empírica acerca de las habilidades adquiridas durante la duración del taller, limitante que deberá ser superada en futuros reportes de investigación.

\section{REFERENCIAS}

Amnistía Internacional (2000). La Zanahoria. México: Amnistía Internacional.

Ayala, A., Carrancedo, G., Hubrad, A., Marín, K., Moreno, O., Pérez, C. \& Santín, M. (2000). Proyecto de Intervención para la promoción y difusión de la escuela para padres. Psicología Iberoamericana, 8, 2, 10-24.

Batres, G. (2000). Manual para el tratamiento grupal del incesto y el abuso sexual infantil. San José, Costa Rica: Programa Regional de Capacitación contra la Violencia Doméstica.

Bond, F. W. \& Dryden, W. (2002). Handbook of Brief Cognitive Behaviour Therapy. Nuava York: John Wiley and Sons, Inc.

Cautela, J. \& Groden, J. (1989). Técnicas de relajación. España: Ediciones Martínez Roca.

Cárdenas, G. (2003). Obstáculos para la educación en derechos humanos en educación primaria. Psicología Iberoamericana, 11, 4, 273-279.

Cascón. P. \& Martín, C. (2000). La alternativa del juego I. Juegos y Dinámicas de educación para la Paz. Madrid: Los libros de la Catarata.
Del Campo, A. \& López, F. (2006). Evaluación de un programa de prevención de abusos sexuales a menores de educación primaria. Psicothema, 18, 1, 1-8.

Echeburúa E. y Guerricaechevarría, C. (1998). Abuso Sexual en la Infancia, en M. A. Vallejo (ed). Manual de Terapia de la conducta, 2. Madrid: Dykinson.

Finkelhor, D. (2005). Abuso sexual al menor. Causas, consecuencias y tratamiento psicosexual. México: Pax.

Hershkowitz, I. Fisher, S., Lamb, M.E., \& Horowitz, D. (2007). Improving Credibility Assessment in Child Sexual Abuse Allegations. The Role of the NICHD Investigative Interview Protocol. Child Abuse and Neglect, 31, 99-110.

Lerman, D. (2003). From the Laboratory to Community Application Translational Research in Behavior Analysis. Journal of Applied Behavior Analysis, $36,4,415-419$.

López, F. (2000). Prevención de los abusos sexuales de menores y educación sexual. Salamanca, España: Amarú Ediciones. 
López, F. y Del Campo, A. (2006). Evaluación de un programa de prevención de abusos sexuales a menores de educación primaria. Psicothema, 18, 1, 1-8.

Martín, M.P. \& Kalus, S. (1978). 1978 Annual Review of child abuse \& Neglect Research. Washington, D.C.: National Center on Child Abuse and Neglect

Mendoza, B. (2002). Intervención en jóvenes víctimas de abusos. Ponencia presentada en el $7^{\circ}$ Congreso Español de Sexología, y Primer Encuentro Iberoamericano de Profesionales de la Sexología. Sevilla, España.

Mendoza, B. (2004a). Validez del testimonio infantil. Ponencia presentada en el VIII Congreso Español de Sexología y II Encuentro Iberoamericano de Profesionales de Sexología. Santander, España.

Mendoza, B. (2004b). Detección de abuso sexual en escuelas de educación básica en la Ciudad de México: Una propuesta de detección grupal. Ponencia presentada en el I Congreso de Violencia Escolar. Brasilia, Brasil.

Nateras, J. O., Torres, J. L \& Linares, M. E. (2004). Ecos de la Consulta Infantil y Juvenil 2000: problemática que preocupa a niños y adolescentes en Tlalpan. Psicología Iberoamericana, 12, 4, 247-255.

Patterson, G. (1982) Coercive Family Process: A Social Learning Approach. Vol. III. Eugene Oregon: Castalia Publishing Co.
Saldivar, G., Ramos, L., Saltijeral, M. T. (2004). Aceptación de la violencia y su relación con los mitos de violación en estudiantes universitarios. Psicología Iberoamericana, 12, 2, 111-121.

Serratos, R. (2003). Repercusiones clínicas y psicológicas del abuso sexual. Ponencia presentada en el VII Congreso Latinoamericano e Iberoamericano sobre la violencia contra niñas, niños y adolescentes, México.

Urra, J. (2002). Tratado de Psicología Forense. Madrid: Siglo XXI.

Vázquez, B. (2003). Abuso sexual infantil. Evaluación de la credibilidad del testimonio. Valencia: Centro Reina Sofía para el estudio de la Violencia.

Whitaker, D. J., Le, B., Hanson, R. K., Baker, Ch. K., McMahon, P. M., Ryan, G. Klein, A., \& Rice, D.(2008). Risks Factors for the Perpetration of Child Sexual Abuse: A Review and Meta Analysis. Child Abuse and Neglect, 32, 529-528.

Young, R. \& Sweeting, H. (2004). Adolescent Bullying, Relations, Psychological Well-Being, and Gender-Atypical Behavior: A Gender Dianosticity Approach. Sex Roles, 50, 7/8, 525-537.

Yuille, J. (1989). Credibility Assessment. Dordrecht. The Netherlands: Kluwer Academic Publisher. 